\title{
Effect of Inflammatory Status on the Association Between Cardiovascular Health and Cognitive Function: A Cross-Sectional Study
}

\section{Ji Heon Lee}

Yonsei University College of Medicine

Ye Jin Jeon

Yonsei University Graduate School

Hyeon Chang Kim

Yonsei University College of Medicine

Sun Jae Jung ( $\square$ sunjaejung@yuhs.ac)

Yonsei University College of Medicine https://orcid.org/0000-0002-5194-7339

\section{Research}

Keywords: cardiovascular risk factors, cognitive decline, immune function

Posted Date: May 12th, 2020

DOI: https://doi.org/10.21203/rs.3.rs-27457/v1

License: (c) (i) This work is licensed under a Creative Commons Attribution 4.0 International License. Read Full License 


\section{Abstract}

Background: Information related to the association between cardiovascular health (CVH) level and cognitive dysfunction in the Korean population is very limited. The objective of our study was to investigate the effect of inflammatory status on the association between $\mathrm{CVH}$ and cognition.

Methods: Data were obtained from the baseline assessments of the Cardiovascular and Metabolic Diseases Etiology Research Center study. Participants who completed fasting serum analysis, questionnaires, and cognitive function tests were included in the analyses, whereas those with a history of autoimmune disease were excluded. Cardiovascular Health in Ambulatory Care Research Team health index metrics, including smoking, physical activity, healthy diet, obesity, history of hypertension, and history of diabetes, were used to assess $\mathrm{CVH}$. Cognitive function was evaluated with the Korean version of the Mini-Mental State Estimation for Dementia Screening. Inflammatory status was assessed based on a high-sensitivity C-reactive protein (hs-CRP) test.

Results: Among 2,622 total participants (mean age 57.2 years; 1,792 women [68.3\%]), 342 (13\%), 1,520 (58\%), and $760(29 \%)$ had poor, intermediate, or ideal CVH, respectively. Logistic regression analysis demonstrated that $\mathrm{CVH}$ was not significantly associated with cognitive function. We further investigated the association between each component of $\mathrm{CVH}$ and low cognitive function by determining the hs-CRP level. Results showed that the only component affecting the association was hypertension history in men.

Conclusions: Our findings suggest that $\mathrm{CVH}$ is not significantly associated with cognitive function in the middle-aged Korean population. In addition, inflammatory status had no significant effect on the association, and the only $\mathrm{CVH}$-related factor affecting the association was hypertension history.

Trial registration: KCT0001038, Clinical Research Information Service (CRIS)

\section{Background}

Dementia has become a serious health problem in the Republic of Korea. In South Korea, the incidence of dementia is almost $10.0 \%$ in the population above age 65 years [1]. The management of dementia in South Korea costs approximately $\$ 200$ million per person, amounting to a total cost of ca. \$16 billion [2]. Moreover, it is a psychosocial burden to both patients and care-givers [3]. Thus, more effective risk management for dementia could address significant economic, psychological, and social problems. For example, early diagnosis of dementia reduces the cost of care for Alzheimer's disease by $\$ 100$ billion annually in the United States [4].

Many previous studies suggested that a lower level of cardiovascular health (CVH) is significantly associated with dementia $[5,6]$. According to prospective cohort studies conducted in the United Kingdom, the dementia incidence rates among participants with a low CVH level were approximately twice that among normal participants [5]. Moreover, a previous study conducted in France showed that an 
increase in optimal $\mathrm{CVH}$ metrics resulting in higher $\mathrm{CVH}$ scores was associated with a lower risk of dementia and cognitive decline [6].

Recently, speculation has increased that inflammatory levels are related to both $\mathrm{CVH}$ and cognitive function. Additionally, numerous research studies have demonstrated that inflammation is associated with the level of $\mathrm{CVH}$. According to a previously published review article, a healthy diet assessed by either a food frequency questionnaire or 24-hour recall, is significantly related to levels of inflammatory markers such as high-sensitivity C-reactive protein (hs-CRP) and interleukin-6 [7]. In addition, physically active individuals display lower levels of inflammatory markers than those with a sedentary lifestyle and increased obesity risk [8]. Obesity may increase the prevalence of cardiometabolic risk factors including glucose intolerance, dyslipidemia, and hypertension [9]. Moreover, other studies reported that the inflammatory response itself was associated with cardiovascular disease [10, 11]. Inflammatory status is not just affected by CVH indicators, but may also be associated with cognition [12]. Moreover, chronic inflammation results in an increase in cytokine levels, incurring hypersecretion of cortisol [11]. Increased steroid levels reduce synthesis of neurotrophic factors and prevent repair of damaged neuronal networks [3]. Furthermore, data from a cohort study of Japanese-American men showed that high levels of hs-CRP increased the risk for all types of dementia [10]. Consequently, based on previously published studies, inflammation level could be considered a possible factor connecting $\mathrm{CVH}$ and cognitive impairment.

Nevertheless, the association between $\mathrm{CVH}$ and cognitive function has not been well-researched in the Korean population. Therefore, in this study we investigated whether inflammatory factors could potentially modify the association between $\mathrm{CVH}$ and cognitive impairment.

\section{Methods}

\section{Study population}

The Cardiovascular and Metabolic Diseases Etiology Research Center (CMERC) study was conducted between 2013-2018 to investigate risk factors for cardiovascular and metabolic disease. The CMERC study enrolled participants meeting the following criteria: (1) aged 30 to 64 years, (2) having an urban residence in Seoul or nearby, (3) able to articulate their own opinions, (4) no history of overt cardiovascular diseases (lifetime), or malignant cancer (within the previous 2 years), (5) no concurrent enrollment in other clinical trials, and (6) not pregnant at baseline. Details of the study are described elsewhere [13]. Cognitive function at age 50 years or older $(N=2,663)$ was evaluated with the Mini-Mental State Examination (MMSE), which was conducted on the entire cohort. Forty-one participants with lifetime autoimmune disease or with incomplete socio-demographic information including education, household income, or marital status were excluded. Finally, a total of 2,622 participants were included in this analysis (Fig. 1). All participants provided written informed consent, and the study protocol was approved by the Yonsei University College of Medicine hospital institutional review board (4-2013-0661). All procedures complied with the ethical standards of the relevant national and institutional committees on human experimentation as per the Helsinki Declaration of 1975 (revised in 2008). 


\section{Assessment of cardiovascular health and risk score}

We used the American Heart Association (AHA) Life's Simple 7 tools (2010) and the Cardiovascular Health in Ambulatory Care Research Team (CANHEART) health index (2013) [14]. Life's Simple 7 tools has an advantage over CANHEART because it includes laboratory test results (total cholesterol and fasting glucose) in addition to healthy lifestyle data (smoking, physical activity, healthy diet, and obesity) [15]. Each of the 7 components were categorized as "poor", "intermediate", or "ideal" according to the AHA's guidelines; details of the criteria applied to each component are described in Supplementary Table 1. The CANHEART health index was developed to evaluate the Canadian population and references data from the Canadian Community Health Survey, a cross-sectional telephone survey of self-reported health status, determinants, and health service use by Canadian adults aged 20 years or older. Unlike Life's Simple 7 tools, the CANHEART health index does not require a laboratory test. The CANHEART health index includes health behaviors such as smoking, physical activity, healthy diet, and obesity, and factors associated with better CVH such as diabetes mellitus and hypertension history [14]. We scored the sum of each component in both cardiovascular health indices, and all were equally weighted (Life's Simple 7 tools score: 0-7; CANHEART health index score: $0-6$ ).

\section{Measurement of cognitive function}

Cognitive function was tested with the Korean version of the Mini Mental State Examination for Dementia Screening (MMSE-DS), administered by trained interviewers to participants aged at least 50 years. The MMSE-DS measures cognitive function using questions to evaluate various categories of cognitive function including time and place, orientation, registration, attention and calculation, memory recall, speaking, and visual construction. If any item had a missing value, the total MMSE-DS score was discarded $(n=6)$. According to a previous validation study in Korea, the test showed excellent internal consistency and diagnostic accuracy (area under the curve, 0.895 [0.880-0.911]) [16].

\section{Measurement of hs-CRP level as an indicator of the inflammatory status}

Inflammatory status was assessed using 8-hour fasting morning blood plasma samples. Plasma levels of hs-CRP were analyzed with a turbid immunoassay (ADVIA1800 Auto Analyzer; Siemens Medical Solutions, Malvern, PA, USA). According to the manufacturer, the detection range for the hs-CRP assay is $0.01-1,000 \mathrm{mg} / \mathrm{L}$, with a sensitivity of $0.2 \mathrm{mg} / \mathrm{L}$.

\section{Covariates}

During the baseline assessment, the participants were asked questions regarding demographic characteristics, socioeconomic status, medical/medication history, family history, and lifestyle factors (smoking, drinking, sleeping, physical activity, and food consumption) by a trained interviewer using a general questionnaire with a standardized protocol. Household income was classified into quartiles (< $24.0,24.0$ to $<34.6,34.6$ to $<49.0$, and $\geq 49.0$ million Korean Won/year). Education level was categorized into groups according to the education curriculum in Korea (elementary school or below, middle school, 
high school, college, or above). Marital status was classified as "never married," "widowed,"

"separated/divorced", or "married and living together". Smoking and drinking were categorized as "never," "past," or "current". Physical activity was assessed with a Korean version of the International Physical Activity Questionnaire-short form, which enquires about the frequency of each of the following activities: walking, moderate-intensity activity, and vigorous activity). The Korean version of the International Physical Activity Questionnaire-short form validity has been confirmed, but has a low reliability [17].

In this study, blood samples were collected after an 8-hour fast and transported on the registration date to the institution where the analysis was performed. Total cholesterol, high-density lipoprotein cholesterol (HDL-C), low-density lipoprotein cholesterol (LDL-C), and triglyceride (TG) levels were measured enzymatically (ADVIA1800 Auto Analyzer; Siemens Medical Solutions, Malvern, PA, USA). Fasting blood glucose levels were measured with a colorimetric assay (ADVIA1800 Auto Analyzer; Siemens Medical Solutions, Malvern, PA, USA).

Height was measured to the nearest $0.1 \mathrm{~cm}$ with an optical linear encoder scale, and body weight was measured to the nearest $0.1 \mathrm{~kg}$ on a digital scale. Body mass index (BMI) was calculated as weight divided by height squared $\left(\mathrm{kg} / \mathrm{m}^{2}\right)$. Upper arm blood pressure was measured three times after the participant had been seated and at rest for at least 5 minutes. We utilized the average of the second and third blood pressure measurements.

\section{Statistical analyses}

Chi-square and analysis of variance (F-test) tests were used to compare baseline differences in covariates of MMSE-DS score categories (cut-off $=24$ ). Continuous variables are shown as mean and standard deviation, while categorical variables are shown as frequency and percentage. A logistic regression model examined the association between the $\mathrm{CVH}$ level and cognitive dysfunction. The final Life's Simple 7 score model was adjusted for age, educational level, household income level, marital status, and current drinking status. We made additional adjustments to the CANHEART health index for mean systolic blood pressure, total cholesterol, and fasting glucose level. The selection of confounders was based on previous studies. Stratified analyses by hs-CRP level were conducted with the same covariates, and we used tertiles to classify the hs-CRP level. Sensitivity analyses were conducted for comparison with pre-imported data using the same logistic regression model $(N=2,241$, Supplementary Table 3), excluding participants who did not complete the dietary questionnaire. All statistical analyses were performed with the SAS 9.4 software (SAS Institute Inc., Cary, NC, USA).

\section{Results}

Characteristics of the study participants $(\mathrm{N}=2,622)$ are shown in Table 1 . Women constituted $68 \%$ of the total study population. The mean age of participants was 57.23 years. Participants were classified by cognitive function as determined by the MMSE-DS score. Based on the MMSE-DS, 87 (3.3\%) participants exhibited low cognitive function (score $<24)$. Lower cognitive function scores were associated with age, 
gender, education level, family income, and smoking, and these factors were adjusted for in the following assessments. 
Table 1

Descriptive characteristics of study participants $(\mathrm{N}=2,622)$

\begin{tabular}{|c|c|c|c|c|c|c|c|}
\hline \multirow[t]{3}{*}{ Variables } & \multirow[t]{3}{*}{ Total } & & \multicolumn{2}{|c|}{$\begin{array}{l}\text { Normal } \\
\text { cognitive } \\
\text { function }\end{array}$} & \multicolumn{2}{|c|}{$\begin{array}{l}\text { Low } \\
\text { cognitive } \\
\text { function }\end{array}$} & \multirow[t]{3}{*}{$\begin{array}{l}\boldsymbol{p} \\
\text { value }\end{array}$} \\
\hline & & & \multicolumn{2}{|c|}{ MMSE-DS $\geq 24$} & \multicolumn{2}{|c|}{ MMSE-DS < 24} & \\
\hline & & & \multicolumn{2}{|c|}{$(n=2,535)$} & \multicolumn{2}{|c|}{$(n=87)$} & \\
\hline Age, years, mean \pm SD & 57.2 & \pm 3.9 & 57.2 & \pm 3.9 & 58.3 & \pm 3.9 & 0.010 \\
\hline \multicolumn{8}{|l|}{ Gender, N(\%) } \\
\hline Men & 830 & (31.7) & 818 & $(32.3)$ & 12 & $(13.8)$ & $<.001$ \\
\hline Women & 1792 & $(68.3)$ & 1,717 & $(67.7)$ & 75 & $(86.2)$ & \\
\hline \multicolumn{8}{|c|}{$\begin{array}{l}\text { Education attainment years, } \\
\mathrm{N}(\%)\end{array}$} \\
\hline$\leq 6$ years & 218 & (8.3) & 192 & (7.6) & 26 & (29.9) & $\begin{array}{l}< \\
0.001\end{array}$ \\
\hline $6-9$ years & 326 & $(12.4)$ & 295 & $(11.6)$ & 31 & $(35.6)$ & \\
\hline $9-12$ years & 1,118 & $(42.6)$ & 1,091 & $(43.0)$ & 27 & (31.0) & \\
\hline $12+$ years & 960 & $(36.6)$ & 957 & $(37.8)$ & 3 & (3.5) & \\
\hline \multicolumn{8}{|c|}{ Family income(year), N(\%) } \\
\hline Q1 & 653 & $(24.9)$ & 612 & $(24.1)$ & 41 & $(47.1)$ & $<0.001$ \\
\hline Q2 & 772 & $(29.4)$ & 743 & $(29.3)$ & 29 & (33.3) & \\
\hline Q3 & 510 & $(19.5)$ & 502 & $(19.8)$ & 8 & $(9.2)$ & \\
\hline Q4 & 687 & $(26.2)$ & 678 & $(26.8)$ & 9 & (10.3) & \\
\hline
\end{tabular}

${ }^{1}$ Participants walking at least 30 minutes per day grouped into regular physical activity group

${ }^{2}$ Self-reported disease history

${ }^{3}$ CANHEART health index and its criteria emphasized by Cardiovascular Health in Ambulatory Care Research Team in 2014

Abbreviation: MMSE-DS, Mini-Mental State Examination-Dementia Screening; SD, Standard Deviation; SBP, Systolic Blood Pressure; DBP, Diastolic Blood Pressure; DM: Diabetes mellitus; HbA1 c:

hemoglobin A1c; hs CRP: high-sensitivity C-reactive protein 


\begin{tabular}{|c|c|c|c|c|c|c|c|}
\hline \multirow[t]{3}{*}{ Variables } & \multirow[t]{3}{*}{ Total } & & \multicolumn{2}{|c|}{$\begin{array}{l}\text { Normal } \\
\text { cognitive } \\
\text { function }\end{array}$} & \multicolumn{2}{|c|}{$\begin{array}{l}\text { Low } \\
\text { cognitive } \\
\text { function }\end{array}$} & \multirow[t]{3}{*}{$\begin{array}{l}p \\
\text { value }\end{array}$} \\
\hline & & & \multicolumn{2}{|c|}{ MMSE-DS $\geq 24$} & \multicolumn{2}{|c|}{ MMSE-DS < 24} & \\
\hline & & & \multicolumn{2}{|c|}{$(n=2,535)$} & \multicolumn{2}{|c|}{$(n=87)$} & \\
\hline Unmarried & 27 & $(1.0)$ & 27 & $(1.1)$ & 0 & $(0.0)$ & 0.330 \\
\hline Married-death of spouse & 151 & $(5.8)$ & 145 & (5.7) & 6 & $(6.9)$ & \\
\hline Married-separated & 146 & $(5.6)$ & 138 & (5.4) & 8 & $(9.2)$ & \\
\hline Married-living together & 2,298 & $(87.6)$ & 2,225 & $(87.8)$ & 73 & $(83.9)$ & \\
\hline \multicolumn{8}{|l|}{ Smoking, N(\%) } \\
\hline Non-smoker & 1,909 & $(72.8)$ & 1,835 & $(72.4)$ & 74 & $(85.1)$ & 0.020 \\
\hline Former smoker & 481 & $(18.3)$ & 470 & $(18.5)$ & 11 & $(12.6)$ & \\
\hline Current smoker & 232 & $(8.9)$ & 230 & (9.1) & 2 & $(2.3)$ & \\
\hline \multicolumn{8}{|l|}{ Drinking, N(\%) } \\
\hline Non-drinker & 670 & $(25.6)$ & 645 & $(25.4)$ & 25 & $(28.7)$ & 0.460 \\
\hline Former drinker & 126 & $(4.8)$ & 124 & $(4.9)$ & 2 & (2.3) & \\
\hline Current drinker & 1,826 & $(69.6)$ & 1,766 & $(69.7)$ & 60 & $(69.0)$ & \\
\hline \multicolumn{8}{|l|}{ Regular physical activity, $\mathrm{N}(\%)^{1}$} \\
\hline No & 705 & $(26.9)$ & 682 & $(26.9)$ & 23 & $(26.4)$ & 0.920 \\
\hline Yes & 1,917 & $(73.1)$ & 1,853 & $(73.1)$ & 64 & $(73.6)$ & \\
\hline Body mass index, mean $\pm S D$ & 24.0 & \pm 2.9 & 24.0 & \pm 2.9 & 24.3 & \pm 2.6 & 0.360 \\
\hline \multicolumn{8}{|l|}{ Ever had hypertension, $\mathrm{N}(\%)^{2}$} \\
\hline No & 2,018 & $(77.0)$ & 1,955 & $(77.1)$ & 63 & $(72.4)$ & 0.310 \\
\hline
\end{tabular}

${ }^{1}$ Participants walking at least 30 minutes per day grouped into regular physical activity group

${ }^{2}$ Self-reported disease history

${ }^{3}$ CANHEART health index and its criteria emphasized by Cardiovascular Health in Ambulatory Care Research Team in 2014

Abbreviation: MMSE-DS, Mini-Mental State Examination-Dementia Screening; SD, Standard Deviation; SBP, Systolic Blood Pressure; DBP, Diastolic Blood Pressure; DM: Diabetes mellitus; HbA1c:

hemoglobin A1c; hs CRP: high-sensitivity C-reactive protein 


\begin{tabular}{|c|c|c|c|c|c|c|c|}
\hline \multirow[t]{3}{*}{ Variables } & \multirow[t]{3}{*}{ Total } & & \multicolumn{2}{|c|}{$\begin{array}{l}\text { Normal } \\
\text { cognitive } \\
\text { function }\end{array}$} & \multicolumn{2}{|c|}{$\begin{array}{l}\text { Low } \\
\text { cognitive } \\
\text { function }\end{array}$} & \multirow[t]{3}{*}{$\begin{array}{l}p \\
\text { value }\end{array}$} \\
\hline & & & \multicolumn{2}{|c|}{ MMSE-DS $\geq 24$} & \multicolumn{2}{|c|}{ MMSE-DS $<24$} & \\
\hline & & & \multicolumn{2}{|c|}{$(n=2,535)$} & \multicolumn{2}{|c|}{$(n=87)$} & \\
\hline Yes & 604 & $(23.0)$ & 580 & $(22.9)$ & 24 & $(27.6)$ & \\
\hline $\mathrm{SBP}, \mathrm{mmHg}$, mean $\pm \mathrm{SD}$ & 120.3 & \pm & 120.4 & \pm 15.2 & 119.2 & \pm 14.3 & 0.480 \\
\hline $\mathrm{DBP}, \mathrm{mmHg}$, mean $\pm \mathrm{SD}$ & 76.6 & \pm 9.6 & 76.6 & \pm 9.6 & 74.7 & \pm 8.3 & 0.060 \\
\hline \multicolumn{8}{|l|}{ Ever had DM, N(\%) ${ }^{2}$} \\
\hline No & 2,438 & $(93.0)$ & 2,359 & $(93.1)$ & 79 & $(90.8)$ & 0.420 \\
\hline Yes & 184 & $(7.0)$ & 176 & $(6.9)$ & 8 & $(9.2)$ & \\
\hline $\begin{array}{l}\text { Fasting insulin, ulU/mL, mean } \pm \\
\text { SD }\end{array}$ & 8.8 & \pm 3.7 & 8.8 & \pm 3.7 & 8.7 & \pm 3.2 & 0.670 \\
\hline $\begin{array}{l}\text { Fasting glusoce, } \mathrm{mg} / \mathrm{dL} \text {, mean } \pm \\
\text { SD }\end{array}$ & 93.9 & $\stackrel{ \pm}{20.3}$ & 93.9 & \pm 20.5 & 93.0 & \pm 14.4 & 0.580 \\
\hline $\mathrm{HbA} 1 \mathrm{c}, \%$, mean $\pm \mathrm{SD}$ & 5.8 & \pm 0.7 & 5.8 & \pm 0.7 & 5.8 & \pm 0.5 & 0.580 \\
\hline hs CRP, mg/L, mean $\pm S D$ & 1.5 & \pm 3.7 & 1.4 & \pm 3.6 & 1.8 & \pm 4.2 & 0.430 \\
\hline \multicolumn{8}{|l|}{ CANHEART health index, $\mathrm{N}(\%)^{3}$} \\
\hline Poor & 342 & $(13.0)$ & 323 & $(12.7)$ & 19 & $(21.8)$ & 0.040 \\
\hline Intermediate & 1,520 & $(58.0)$ & 1,477 & $(58.3)$ & 43 & $(49.4)$ & \\
\hline Ideal & 760 & $(29.0)$ & 735 & $(29.0)$ & 25 & $(28.7)$ & \\
\hline \multicolumn{8}{|c|}{${ }^{1}$ Participants walking at least 30 minutes per day grouped into regular physical activity group } \\
\hline \multicolumn{8}{|l|}{${ }^{2}$ Self-reported disease history } \\
\hline \multicolumn{8}{|c|}{$\begin{array}{l}3 \text { CANHEART health index and its criteria emphasized by Cardiovascular Health in Ambulatory Care } \\
\text { Research Team in } 2014\end{array}$} \\
\hline \multicolumn{8}{|c|}{$\begin{array}{l}\text { Abbreviation: MMSE-DS, Mini-Mental State Examination-Dementia Screening; SD, Standard Deviation; } \\
\text { SBP, Systolic Blood Pressure; DBP, Diastolic Blood Pressure; DM: Diabetes mellitus; HbA1c: } \\
\text { hemoglobin A1c; hs CRP: high-sensitivity C-reactive protein }\end{array}$} \\
\hline
\end{tabular}

\section{CANHEART health index and cognitive function}


Tables 2 and 3 represent the association between CVH (evaluated by CANHEART health index, poor $=0-1$, intermediate $=2-5$, and ideal $=6$ ) and cognitive decline (evaluated by MMSE-DS, score $<24$ ) in men and women, respectively. A stratified analysis across hs-CRP tertiles was conducted with the same data. There was no significant association between $\mathrm{CVH}$ and cognitive dysfunction in men or women. There were no statistically meaningful associations in the stratified analysis conducted across hs-CRP tertiles. In Supplementary Table 2, we assessed the association between Life's Simple 7 score (poor $=0-2$, intermediate $=3-4$, ideal $=5-7$ ) and cognitive decline for comparison. Poor CVH measured by Life's Simple 7 score positively associated with low cognitive function than ideal $\mathrm{CVH}$, but not statistically significant (men, odds ratio: 1.71, 95\% Cl: 0.37-7.93; women, odds ratio: 1.20, 95\% Cl: 0.55-2.58). 


\section{CANHEART Low cognitive function (MMSE-DS < 24)}

health index ${ }^{1}$
Age adjusted Model 1, additionally adjusted model for SES and drinking status

$\mathrm{OR}^{2} \quad\left(95 \% \quad \mathrm{OR}^{2} \quad(95 \% \mathrm{Cl})\right.$
Model 2, additionally adjusted for health status

$\mathrm{OR}^{2} \quad(95 \% \mathrm{Cl})$

\section{Total men}

$(\mathrm{N}=830)$

$\begin{array}{ccccccc}\begin{array}{c}\text { Poor } \\ (\mathrm{n}=274)\end{array} & 8.25 & \begin{array}{l}(0.47- \\ 145.40)\end{array} & 7.07 & (0.56-88.93) & 9.33 & (0.76-14.13) \\ \begin{array}{c}\text { Intermediate } \\ (\mathrm{n}=279)\end{array} & 4.28 & \begin{array}{c}(0.25- \\ 72.61)\end{array} & 4.62 & (0.38-55.90) & 5.61 & (0.49-64.87) \\ \begin{array}{c}\text { Ideal } \\ (\mathrm{n}=277)\end{array} & 1.00 & \text { ref } & 1.00 & \text { ref } & 1.00 & \text { ref }\end{array}$

\section{Stratified analysis by hs-CRP}

\section{hs-CRP, lower}

tertile

$\begin{array}{lcllcll}\begin{array}{l}\text { Poor } \\ (\mathrm{n}=41)\end{array} & 11.68 & \begin{array}{l}(0.64- \\ 212.35)\end{array} & 9.06 & (0.65-126.07) & 14.59 & (0.94-27.17) \\ \begin{array}{l}\text { Intermediate } \\ (\mathrm{n}=168)\end{array} & 1.95 & \begin{array}{l}(0.10- \\ 37.46)\end{array} & 3.86 & (0.27-55.28) & 5.40 & (0.36-80.16) \\ & & & & & \end{array}$

$\begin{array}{ccccccc}\begin{array}{c}\text { Ideal } \\ (n=65)\end{array} & 1.00 & \text { ref } & 1.00 & \text { ref } & 1.00 & \text { ref }\end{array}$

\section{hs-CRP,}

middle tertile

$\begin{array}{ccccccc}\begin{array}{c}\text { Poor } \\ (n=65)\end{array} & 0.50 & \begin{array}{c}(0.01- \\ 23.13)\end{array} & 0.58 & (0.03-10.43) & 0.83 & (0.09-7.39) \\ \begin{array}{c}\text { Intermediate } \\ (n=166)\end{array} & 1.30 & \begin{array}{l}(0.07- \\ 24.86)\end{array} & 1.74 & (0.19-16.00) & 1.28 & (0.22-7.39) \\ \begin{array}{c}\text { Ideal } \\ (n=48)\end{array} & 1.00 & \text { ref } & 1.00 & \text { ref } & 1.00 & \text { ref }\end{array}$

\section{hs-CRP, higher tertile}

\begin{tabular}{lcccccc}
$\begin{array}{c}\text { Poor } \\
(n=78)\end{array}$ & 1.71 & $\begin{array}{c}(0.08- \\
35.79)\end{array}$ & 1.15 & $(0.08-17.34)$ & 1.04 & $(0.08-14.36)$ \\
$\begin{array}{l}\text { Intermediate } \\
(n=171)\end{array}$ & 1.12 & $\begin{array}{l}(0.06- \\
21.60)\end{array}$ & 0.88 & $(0.06-12.32)$ & 0.86 & $(0.07-10.55)$ \\
\hline & & & & & &
\end{tabular}




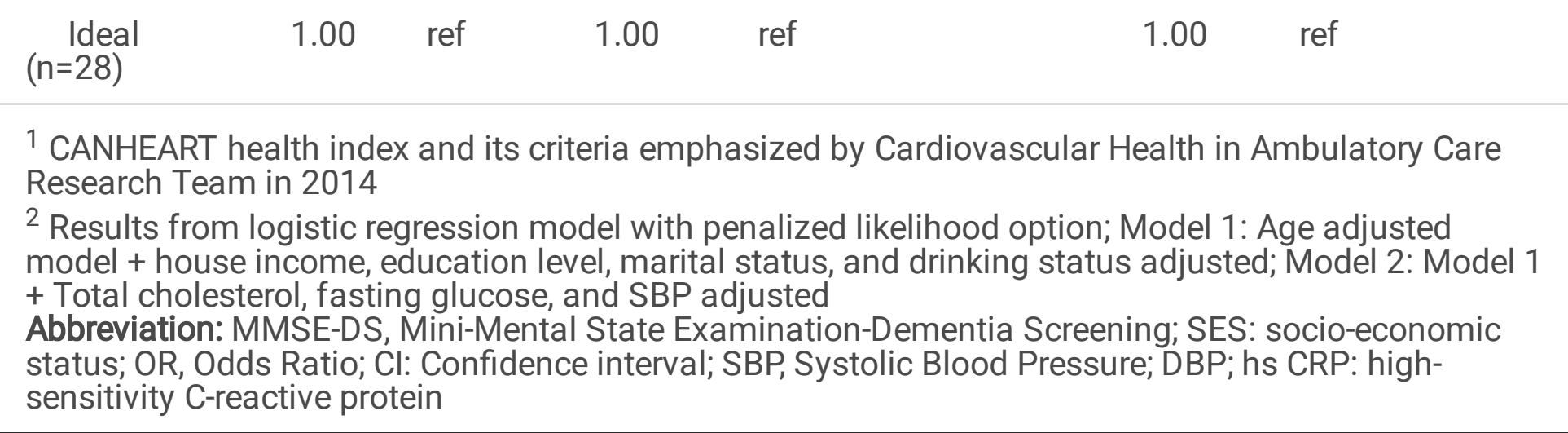

Table 2

Association between CANHEART health index and cognitive function in men by hs CRP tertile $(\mathrm{N}=830)$ 
CANHEART Low cognitive function (MMSE-DS < 24)

health index ${ }^{1}$

Age adjusted Model 1, additionally adjusted for model

$\mathrm{OR}^{2} \quad\left(95 \% \quad \mathrm{OR}^{2} \quad(95 \% \mathrm{Cl})\right.$

$\mathrm{Cl})$
SES and drinking status
Model 2, additionally adjusted for health status

$\mathrm{OR}^{2} \quad(95 \% \mathrm{Cl})$

Total women $(\mathrm{N}=1,792)$

\begin{tabular}{ccccccc}
$\begin{array}{c}\text { Poor } \\
(n=158)\end{array}$ & 2.37 & $\begin{array}{l}(1.27- \\
4.45)\end{array}$ & 1.61 & $(0.84-3.09)$ & 2.03 & $(1.002-4.11)$ \\
$\begin{array}{c}\text { Intermediate } \\
(n=1,015)\end{array}$ & 0.96 & $\begin{array}{l}(0.58- \\
1.59)\end{array}$ & 0.76 & $(0.45-1.28)$ & 0.83 & $(0.49-1.41)$ \\
$\begin{array}{c}\text { Ideal } \\
(n=619)\end{array}$ & 1.00 & ref & 1.00 & ref & 1.00 & ref \\
\hline
\end{tabular}

\section{Stratified analysis by hs-CRP}

\section{hs-CRP, lower}

tertile

$\begin{array}{ccccccc}\text { Poor }(\mathrm{n}=24) & 3.55 & \begin{array}{l}(1.03- \\ 12.27)\end{array} & 2.81 & (0.79-9.96) & 2.27 & (0.62-8.37) \\ \begin{array}{c}\text { Intermediate } \\ (\mathrm{n}=304)\end{array} & 1.26 & \begin{array}{l}(0.52- \\ 3.02)\end{array} & 1.00 & (0.43-2.37) & 0.93 & (0.39-2.20) \\ \begin{array}{c}\text { Ideal } \\ (\mathrm{n}=256)\end{array} & 1.00 & \text { ref } & 1.00 & \text { ref } & 1.00 & \text { ref }\end{array}$

\section{hs-CRP, middle}

tertile

$\begin{array}{lllllll}\text { Poor }(\mathrm{n}=44) & 3.36 & \begin{array}{l}(1.16- \\ 9.67)\end{array} & 2.17 & (0.70-6.67) & 3.44 & (0.93-12.73) \\ & 0.55 & \begin{array}{l}(0.22- \\ 1.34)\end{array} & 0.38 & (0.15-0.95) & 0.43 & (0.17-1.10)\end{array}$

$(n=349)$

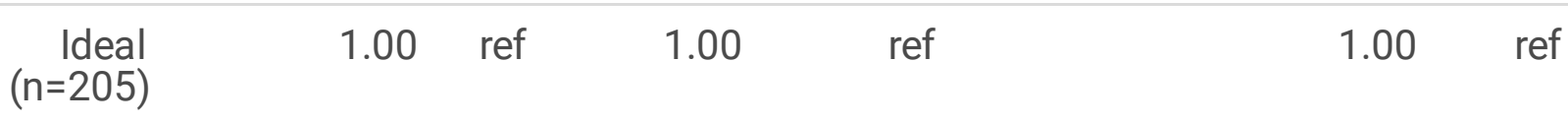

\section{hs-CRP, higher \\ tertile}

$\begin{array}{lllllll}\text { Poor }(\mathrm{n}=80) & 1.15 & \begin{array}{l}(0.35- \\ 3.82)\end{array} & 0.81 & (0.25-2.69) & 1.16 & (0.32-4.17) \\ & 0.87 & \begin{array}{l}(0.35- \\ 2.13)\end{array} & 0.68 & (0.28-1.68) & 0.81 & (0.32-2.04) \\ \text { termediate } & & & & & \end{array}$




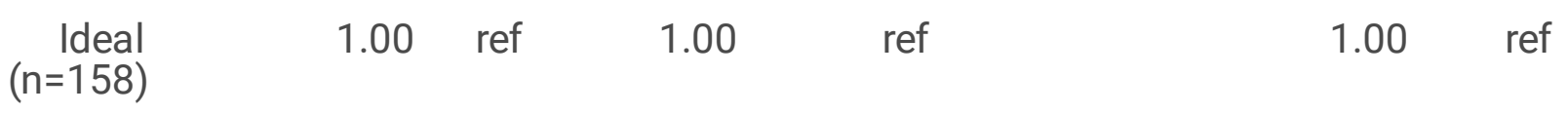

${ }^{1}$ CANHEART health index and its criteria emphasized by Cardiovascular Health in Ambulatory Care Research Team in 2014

${ }^{2}$ Results from logistic regression model with penalized likelihood option; Model 1: Age adjusted model + house income, education level, marital status, and drinking status adjusted; Model 2: Model 1 + Total cholesterol, fasting glucose, and SBP adjusted Abbreviation: MMSE-DS, Mini-Mental State Examination-Dementia Screening; SES: socio-economic status; OR, Odds Ratio; Cl: Confidence interval; SBP, Systolic Blood Pressure; DBP; hs CRP: highsensitivity C-reactive protein

Table 3

Association between CANHEART health index and cognitive function in women by hs CRP tertile $(\mathrm{N}=1,792)$

\section{Components of CANHEART health index and cognitive function}

Tables 4 and 5 show results on the association of each component of the CANHEART health index, including current smoking status, overweight status, leisure physical activity, fruit and vegetable consumption, hypertension, and diabetes, with cognitive decline in men and women, respectively. Low cognitive function (odds ratio: $3.79,95 \% \mathrm{Cl}$ : 1.29-11.1) was associated with hypertension in men. However, there was no significant association between the CANHEART health index components and low cognitive function in women. 
CANHEART health index $^{1}$ (each components)
Cognitive dysfunction (MMSE-DS < 24)

\begin{tabular}{|c|c|c|c|c|c|c|}
\hline Total & & $\begin{array}{l}\text { hs-C } \\
\text { terti }\end{array}$ & lower & $\begin{array}{l}\text { hs-CF } \\
\text { midd }\end{array}$ & tertile & $\begin{array}{l}\text { hs-C } \\
\text { terti }\end{array}$ \\
\hline $\mathrm{OR}^{2}$ & $\begin{array}{l}(95 \% \\
\mathrm{Cl})\end{array}$ & $\mathrm{OR}^{2}$ & $\begin{array}{l}(95 \% \\
\mathrm{Cl})\end{array}$ & $\mathrm{OR}^{2}$ & $\begin{array}{l}(95 \% \\
\mathrm{Cl})\end{array}$ & $\mathrm{OR}^{2}$ \\
\hline
\end{tabular}

(1) Smoking

\begin{tabular}{lclllllll} 
Current smoker $(\mathrm{n}=233)$ & 0.70 & $\begin{array}{l}(0.23- \\
2.09)\end{array}$ & 1.01 & $\begin{array}{l}(0.19- \\
0.30)\end{array}$ & 0.65 & $\begin{array}{l}(0.14 \\
-3.01)\end{array}$ & 0.59 & $\begin{array}{l}(0.14- \\
2.41)\end{array}$ \\
$\begin{array}{c}\text { Nonsmoker or former } \\
(n=597)\end{array}$ & 1.00 & ref & 1.00 & ref & 1.00 & ref & 1.00 & ref \\
\hline
\end{tabular}

(2) Overweight, obesity

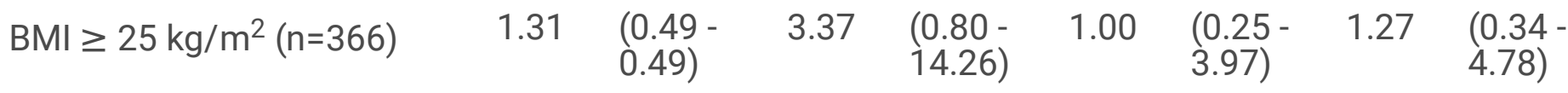

$\mathrm{BMI}<25 \mathrm{~kg} / \mathrm{m}^{2}(\mathrm{n}=464) \quad 1.00$ ref $\quad 1.00$ ref $\quad 1.00$ ref 1.00 ref

(3) Leisure physical activity

$<30$ min per day $(n=218)$
$\begin{array}{lllll}1.27 & (0.45- & 0.52 & (0.09- & 0.73\end{array}$
(0.15- 3.62)
2.47
$(0.68-$ $3.57)$
3.02) 9.04)

$\geq 30$ min per day $(n=612)$

1.00 ref

1.00 ref

1.00 ref

1.00 ref

(4) Fruit and vegetable consumption

\begin{tabular}{|c|c|c|c|c|c|c|c|c|}
\hline $\begin{array}{l}<5 \text { servings per day } \\
(\mathrm{n}=241)\end{array}$ & 2.04 & $\begin{array}{l}(0.75 \\
5.53)\end{array}$ & 0.96 & $\begin{array}{l}(0.21- \\
4.47)\end{array}$ & 1.49 & $\begin{array}{l}(0.37- \\
5.97)\end{array}$ & 3.83 & $\begin{array}{l}(0.91 \\
16.13\end{array}$ \\
\hline $\begin{array}{c}\geq 5 \text { servings per day } \\
(\mathrm{n}=589)\end{array}$ & 1.00 & ref & 1.00 & ref & 1.00 & ref & 1.00 & ref \\
\hline
\end{tabular}

(5) Hypertension ${ }^{3}$

$\begin{array}{lllllllll}\text { Yes }(\mathrm{n}=240) & 3.79 & (1.29- & 7.68 & (1.66- & 0.88 & (0.18- & 1.37 & (0.35- \\ & & 11.10) & & 35.59) & & 4.30) & & 5.34) \\ \text { No }(n=590) & 1.00 & \text { ref } & 1.00 & \text { ref } & 1.00 & \text { ref } & 1.00 & \text { ref }\end{array}$

(6) Diabetes $^{3}$

$\begin{array}{lllllllll}\text { Yes }(n=87) & 1.73 & \begin{array}{l}(0.37- \\ 8.23)\end{array} & 4.81 & \begin{array}{l}(0.47- \\ 49.71)\end{array} & 4.40 & \begin{array}{c}(0.80- \\ 24.15)\end{array} & 0.55 & (0.04- \\ \text { No }(n=743) & 1.00 & \text { ref } & 1.00 & \text { ref } & 1.00 & \text { ref } & 1.00 & \text { ref }\end{array}$

${ }^{1}$ CANHEART health index and its criteria emphasized by Cardiovascular Health in Ambulatory Care Research Team in 2014

${ }^{2}$ Results from logistic regression model with penalized likelihood option, adjusted for age, education level, household income, marital status, current drinking status, total cholesterol, fasting glucose level, 
mean SBP

${ }^{3}$ Self-reported disease history (lifetime)

Abbreviation: MMSE-DS, Mini-Mental State Examination-Dementia Screening; OR, Odds Ratio; Cl: Confidence interval; SBP, Systolic Blood Pressure; DBP; hs CRP: high-sensitivity C-reactive protein

Table 4

Association between each CANHEART health index component and cognitive function in men by hs CRP tertiles $(\mathrm{N}=830)$ 
CANHEART health index ${ }^{1}$

(each components)
Cognitive dysfunction (MMSE-DS < 24)

\begin{tabular}{|c|c|c|c|c|c|c|}
\hline Total & & $\begin{array}{l}\text { hs } \mathrm{Cl} \\
\text { tertile }\end{array}$ & lower & $\begin{array}{l}\text { hs C } \\
\text { tertil }\end{array}$ & middle & $\begin{array}{l}\text { hs CRP, } \\
\text { higher tertile }\end{array}$ \\
\hline $\mathrm{OR}^{2}$ & $\begin{array}{l}(95 \% \\
\mathrm{Cl})\end{array}$ & $\mathrm{OR}^{2}$ & $(95 \% \mathrm{Cl})$ & $\mathrm{OR}^{2}$ & $\begin{array}{l}(95 \% \\
\mathrm{Cl})\end{array}$ & $\begin{array}{ll}\mathrm{OR}^{2} & (95 \% \\
\mathrm{Cl})\end{array}$ \\
\hline
\end{tabular}

(1) Smoking

$\begin{array}{lllllllll}\text { Current smoker }(\mathrm{n}=26) & 0.25 & \begin{array}{l}(0.02- \\ 4.27)\end{array} & 0.92 & (0.04- & 1.51 & (0.08- & 0.26 & (0.01 \\ & & & & 23.50) & & 29.72) & & - \\ & & & & & & & & \end{array}$

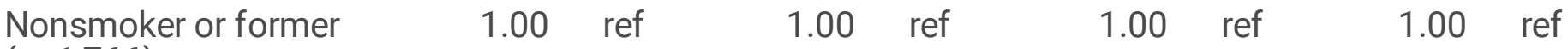
$(n=1,766)$

(2) Overweight, obesity

$\begin{array}{lllllllll}\mathrm{BMI} \geq 25 \mathrm{~kg} / \mathrm{m}^{2}(\mathrm{n}=520) & 1.28 & \begin{array}{l}(0.78- \\ 2.11)\end{array} & 2.05 & \begin{array}{l}(0.86- \\ 4.88)\end{array} & 0.96 & (0.41- & 1.25 & (0.56 \\ & & & & & & & & 2.24) \\ & & & & & & \\ \mathrm{BMI}<25 \mathrm{~kg} / \mathrm{m}^{2}(\mathrm{n}=1,272) & 1.00 & \text { ref } & 1.00 & \text { ref } & 1.00 & \text { ref } & 1.00 & \text { ref }\end{array}$

(3) Leisure physical activity

\begin{tabular}{|c|c|c|c|c|c|c|c|c|}
\hline$<30$ min per day $(n=487)$ & 1.02 & $\begin{array}{l}(0.60- \\
1.73)\end{array}$ & 0.98 & $\begin{array}{l}(0.40- \\
2.36)\end{array}$ & 1.03 & $\begin{array}{l}(0.40- \\
2.65)\end{array}$ & 1.30 & $\begin{array}{l}(0.55 \\
- \\
3.06)\end{array}$ \\
\hline$\underset{(n=1,305)}{\geq 30 \text { min per day }}$ & 1.00 & ref & 1.00 & ref & 1.00 & ref & 1.00 & ref \\
\hline
\end{tabular}

(4) Fruit and vegetable consumption

$\begin{array}{lllllllll}\begin{array}{l}<5 \text { servings per day } \\ (n=370)\end{array} & 1.44 & \begin{array}{l}(0.85- \\ 2.44)\end{array} & 1.43 & \begin{array}{c}(0.58- \\ 3.52)\end{array} & 2.52 & \begin{array}{c}(1.06- \\ 6.00)\end{array} & 0.89 & \begin{array}{l}(0.35 \\ 2.28)\end{array} \\ \begin{array}{l}\geq 5 \text { servings per day } \\ (n=1,422)\end{array} & 1.00 & \text { ref } & 1.00 & \text { ref } & 1.00 & \text { ref } & 1.00 & \text { ref }\end{array}$

(5) Hypertension ${ }^{3}$

\begin{tabular}{|c|c|c|c|c|c|c|c|}
\hline Yes $(n=364)$ & 0.93 & $\begin{array}{l}(0.52- \\
1.65)\end{array}$ & 1.37 & $\begin{array}{l}(0.54- \\
3.49)\end{array}$ & 0.65 & $\begin{array}{l}(0.22- \\
1.92)\end{array}$ & 0.84 \\
\hline
\end{tabular}

No $(n=1,428) \quad 1.00 \quad$ ref $\quad 1.00 \quad$ ref $\quad 1.00 \quad$ ref $\quad 1.00$ ref

(6) Diabetes $^{3}$

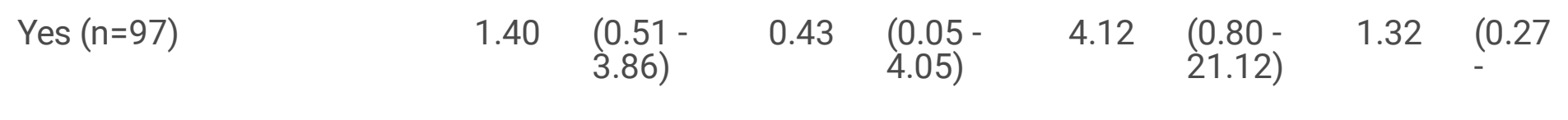




No $(n=1,695) \quad 1.00$ ref $\quad 1.00$ ref $\quad 1.00$ ref $\quad 1.00$ ref

${ }^{1}$ CANHEART health index and its criteria emphasized by Cardiovascular Health in Ambulatory Care Research Team in 2014

${ }^{2}$ Results from logistic regression model with penalized likelihood option, adjusted for age, education level, household income, marital status, current drinking status, total cholesterol, fasting glucose level, mean SBP

${ }^{3}$ Self-reported disease history (lifetime)

Abbreviation: MMSE-DS, Mini-Mental State Examination-Dementia Screening; OR, Odds Ratio; Cl: Confidence interval; SBP, Systolic Blood Pressure; DBP; hs CRP: high-sensitivity C-reactive protein

Table 5

Association between each CANHEART health index component and cognitive function in women by hs CRP tertiles $(\mathrm{N}=1,792)$

\section{Discussion}

The results of this study demonstrated that the association between $\mathrm{CVH}$ and cognitive function was not significant, and that the level of hs-CRP did not affect the risk of cognitive dysfunction. CVH assessment results obtained using the CANHEART health index did not show an association with cognition in either men or women. A stratified analysis across hs-CRP tertiles revealed no association between hs-CRP level and cognitive function. Although the presence of hypertension in men was associated with cognitive dysfunction, no other component had a significant association with cognition in either men or women.

Several explanations for these results may be suggested. First, the relatively small number of participants in the low cognitive function group should be considered. In this study, only 87 participants of the 2,622 total study population had an MMSE score below 24. The number of participants with low cognitive function in this study was relatively small compared to that in previous studies. A previous research study conducted in France included 745 dementia patients in a total study population of 6,626 [6]. Another study conducted in the UK included 347 dementia patients in a total population of 7,899 [5]. Consequently, the smaller sizes of the low cognitive function group and total population in our study could have lowered the accuracy of the analysis.

Second, some factors may have contributed to higher cognitive function in our study participants compared to that in previous studies, notably, the differences between the study populations. For example, the fruit and vegetable consumption in Korea is $452 \mathrm{~g}$ per day [18], whereas that in France is $342 \mathrm{~g} /$ day and in the UK is $258 \mathrm{~g} /$ day [19]. Comparatively high consumption of fruits and vegetables could contribute to a delay in cognitive decline [20]. It is possible that unknown differences in Eastern/Western population health-related distribution might have an effect. In addition, application of different criteria for measuring cognitive decline in the studies could lead to differences in results. In our study, the criteria for cognitive decline included mild cognitive impairment (MCl), whereas the criterion in previous studies was dementia $[5,6]$. Since $\mathrm{MCl}$ is a pre-dementia stage and its severity is less than that of dementia [21,22], the correlation between $\mathrm{CVH}$ and $\mathrm{MCl}$ and that between $\mathrm{CVH}$ and dementia could be 
different. Differences in the methods for measuring cognitive dysfunction could be another reason for inconsistent study outcomes. In our study, the Korean version of the MMSE-DS was used, whereas previous studies used a combination of cognitive tests [5].

Additionally, the differences between the AHA's Life's Simple 7 tools and the CANHEART model might also cause dissimilar results $[23,24]$. The AHA's Life's Simple 7 tools includes smoking, physical activity, healthy diet, BMI, total cholesterol, blood pressure, and fasting plasma glucose [14, 23, 24]. In comparison, the CANHEART index includes smoking, leisure physical activity, fruit and vegetable consumption, BMI, hypertension, and diabetes; several of these components are subjective and may be easily affected by recall bias [25]. Consequently, the participant CVH level could be underestimated when using the CANHEART components. In this case, participants with normal CVH levels could be classified as having low $\mathrm{CVH}$ level, making the relationship between low $\mathrm{CVH}$ and low cognitive function appear weaker. Compared to the CANHEART model, the AHA's Life's Simple 7 tools includes objective components, such as total cholesterol and fasting plasma glucose levels; therefore, the influence of recall bias in previous studies could be less than that observed in our study.

The current study has implications for the design of future studies. Primarily, our study and the previous studies obtained very different results. We found that CVH levels did not show an association with cognitive function in our study population. It is still unknown whether the differences in results were related to statistics or differences between Korean and European populations. The current study also confirmed that inflammation levels had no significant association with cognitive dysfunction. This will potentially simplify future study designs.

Our study had several limitations. First, the CANHEART model used for measuring CVH has a low applicability. This model was developed in Canada and has been applied extremely infrequently elsewhere. Second, our study had a cross-sectional design; therefore, the temporal association between $\mathrm{CVH}$ and cognitive function could not be verified. Third, the elements that may have caused conflicting results in studies investigating associations between $\mathrm{CVH}$ and cognitive function in different populations are unknown. Several factors such as genetic differences between races [26], degree of fruit and vegetable consumption $[27,28]$, or environmental and cultural influences could be relevant. Further investigations to develop an appropriate index for the Korean population are needed.

In conclusion, our study revealed that the association between low CVH levels and cognitive decline was not significant and that inflammation levels were not relevant to cognitive decline. Several recommendations can be made for designing future studies to ascertain why our results conflicted with those of previous studies. A larger study population is important, since in our study, the low number of participants was potentially the cause of low significance. In addition, the AHA's Life's Simple 7 model is cautiously recommended to reduce potential subjectivity. The methods used to evaluate cognitive function in future studies should be similar to those used in previous studies, allowing accurate comparisons. Consequently, efforts to resolve the issue of conflicting outcomes between this study and previous studies could be focused on for distinguishing the characteristics of the study populations. 


\section{Abbreviations}

CVH: cardiovascular health

hs-CRP: high-sensitivity C-reactive protein

CMERC: Cardiovascular and Metabolic Diseases Etiology Research Center

MMSE: Mini-Mental State Examination

AHA: American Heart Association

CANHEART: Cardiovascular Health in Ambulatory Care Research Team

MCl: mild cognitive impairment

\section{Declarations}

\section{Ethics approval and consent to participate:}

The authors assert that all procedures contributing to this work comply with the ethical standards of the relevant national and institutional committees on human experimentation, and with the Helsinki Declaration of 1975, as revised in 2008.

\section{Consent for publication:}

Not applicable

\section{Availability of data and materials:}

The datasets used and/or analyzed during the current study are available from the corresponding author on reasonable request.

\section{Competing interests:}

The authors declare that they have no competing interests

\section{Funding:}

This research was supported by the Basic Science Research Program through the National Research Foundation of Korea (NRF) funded by the Ministry of Science and ICT (2018R1C1B5083722, 


\section{Authors' contributions}

Dr. Jung had full access to all of the data in the study and takes responsibility for the integrity of the data and the accuracy of the data analysis.

Study design and concept: Jung, Lee, Jeon

Acquisition, analysis, or interpretation of data: Jung, Lee, Jeon

Drafting of the manuscript: Lee

Critical revision of the manuscript for important intellectual content: Jung, Jeon, Kim

Statistical analysis: Jeon, Lee

Administrative, technical, or material support: Lee, Jeon

Obtained funding and study supervision: Jung

\section{Acknowledgements:}

Not applicable

\section{References}

1. Kim YJ, Han JW, So YS, Seo JY, Kim KY, Kim KW. Prevalence and trends of dementia in Korea: a systematic review and meta-analysis. J Korean Med Sci. 2014;29(7):903-12. doi:10.3346/jkms.2014.29.7.903.

2. Kim K, Kim H, Ahn H, Kim Y, Whang J, Kim B. Korean dementia observatory 2016. National Institute of Dementia Report. Seongnam-si: National Institute of Dementia; 2016 December. Report No. In: NIDR-1603-0015.

3. Brodaty H. Psychosocial Effects on Carers of Living with Persons with Dementia. 1990.

4. Leifer BP. Early diagnosis of Alzheimer's disease: clinical and economic benefits. J Am Geriatr Soc. 2003;51(5 Suppl Dementia):S281-8. doi:10.1046/j.1532-5415.5153.x.

5. Sabia S, Fayosse A, Dumurgier J, Schnitzler A, Empana JP, Ebmeier KP, et al. Association of ideal cardiovascular health at age 50 with incidence of dementia: 25 year follow-up of Whitehall II cohort study. BMJ. 2019;366:I4414. doi:10.1136/bmj.14414.

6. Samieri C, Perier MC, Gaye B, Proust-Lima C, Helmer C, Dartigues JF, et al. Association of Cardiovascular Health Level in Older Age With Cognitive Decline and Incident Dementia. JAMA. 
2018;320(7):657-64. doi:10.1001/jama.2018.11499.

7. L. G. Diet and inflammation. 2010.

8. Ertek SaAC. Impact of physical activity on inflammation: effects on cardiovascular disease risk and other inflammatory conditions. 2012.

9. Monteiro RalA. Chronic inflammation in obesity and the metabolic syndrome. 2010.

10. Schmidt R, Schmidt H, Curb JD, Masaki K, White LR, Launer LJ. Early inflammation and dementia: a 25-year follow-up of the Honolulu-Asia Aging Study. Ann Neurol. 2002;52(2):168-74.

doi:10.1002/ana.10265.

11. Leonard B. Inflammation, Depression and Dementia: Are they Connected? 2007.

12. Sartori AC. The impact of inflammation on cognitive function in older adults: implications for healthcare practice and research. 2012.

13. Shim JS, Song BM, Lee JH, Lee SW, Park JH, Choi DP, et al. Cardiovascular and Metabolic Diseases Etiology Research Center (CMERC) cohort: study protocol and results of the first 3 years of enrollment. Epidemiol Health. 2017;39:e2017016. doi:10.4178/epih.e2017016.

14. Maclagan LC, Park J, Sanmartin C, Mathur KR, Roth D, Manuel DG, et al. The CANHEART health index: a tool for monitoring the cardiovascular health of the Canadian population. Cmaj. 2014;186(3):180-7. doi:10.1503/cmaj.131358.

15. Lloyd-Jones DM, Hong Y, Labarthe D, Mozaffarian D, Appel LJ, Van Horn L, et al. Defining and setting national goals for cardiovascular health promotion and disease reduction: the American Heart Association's strategic Impact Goal through 2020 and beyond. Circulation. 2010;121(4):586-613. doi:10.1161/circulationaha.109.192703.

16. Kim TH, Jhoo JH, Park JH, Kim JL, Ryu SH, Moon SW, et al. Korean version of mini mental status examination for dementia screening and its' short form. Psychiatry Investig. 2010;7(2):102-8. doi:10.4306/pi.2010.7.2.102.

17. Chun MY. Validity and reliability of korean version of international physical activity questionnaire short form in the elderly. Korean J Fam Med. 2012;33(3):144-51. doi:10.4082/kjfm.2012.33.3.144.

18. Statista. Annual consumption volume of vegetables per capita in South Korea from 2011 to 2018. 2018.

19. Authority EFS. Concise Database summary statistics-total population. 2008.

20. Loef $M$, Walach $H$. Fruit, vegetables and prevention of cognitive decline or dementia: a systematic review of cohort studies. J Nutr Health Aging. 2012;16(7):626-30. doi:10.1007/s12603-012-0097-x.

21. T.Gabryelewicz. The rate of conversion of mild cognitive impairment to dementia: predictive role of depression. 2006.

22. Panza F. Late-Life Depression, Mild Cognitive Impairment and Dementia: Possible Continuum? 2010.

23. Folsom AR, Shah AM, Lutsey PL, Roetker NS, Alonso A, Avery CL, et al. American Heart Association's Life's Simple 7: Avoiding Heart Failure and Preserving Cardiac Structure and Function. Am J Med. 2015;128(9):970-6 e2. doi:10.1016/j.amjmed.2015.03.027. 
24. Goff DC, Lloyd-Jones DM, Bennett G, Coady S, D’Agostino RB, Gibbons R, et al. 2013 ACC/AHA Guideline on the Assessment of Cardiovascular Risk. Journal of the American College of Cardiology. 2014;63(25):2935-59. doi:10.1016/j.jacc.2013.11.005.

25. Jordana K Schmier MTH. Patient recall and recall bias of health state and health status. 2014.

26. Hackler E, 3rd, Lew J, Gore MO, Ayers CR, Atzler D, Khera A, et al. Racial Differences in Cardiovascular Biomarkers in the General Population. J Am Heart Assoc. 2019;8(18):e012729. doi:10.1161/jaha.119.012729.

27. Stearns JC, Zulyniak MA, de Souza RJ, Campbell NC, Fontes M, Shaikh M, et al. Ethnic and dietrelated differences in the healthy infant microbiome. Genome Med. 2017;9(1):32. doi:10.1186/s13073-017-0421-5.

28. Li W, Youssef G, Procter-Gray E, Olendzki B, Cornish T, Hayes R, et al. Racial Differences in Eating Patterns and Food Purchasing Behaviors among Urban Older Women. J Nutr Health Aging. 2017;21(10):1190-9. doi:10.1007/s12603-016-0834-7.

\section{Figures}

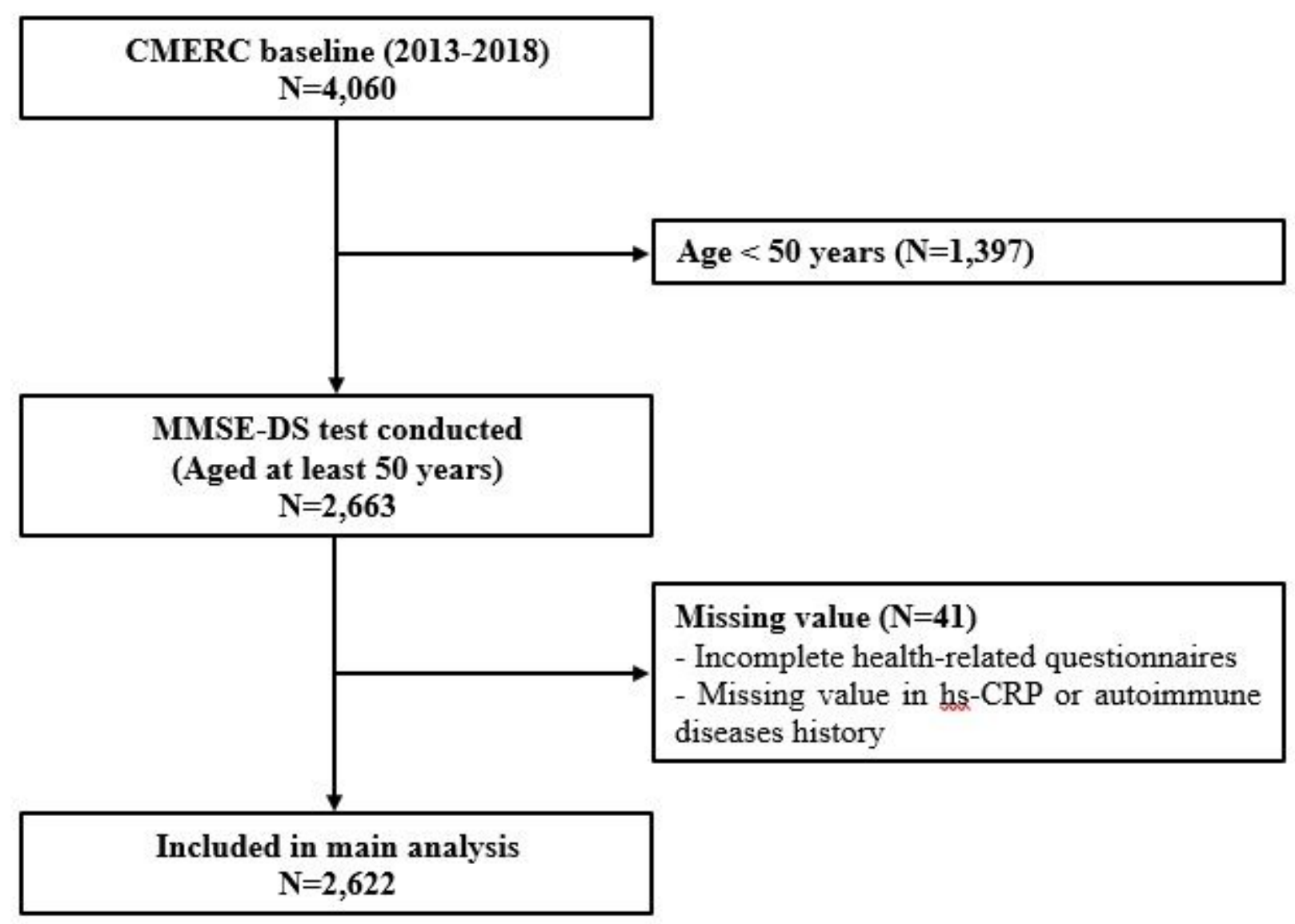

Figure 1 
Flowchart of the study participants

\section{Supplementary Files}

This is a list of supplementary files associated with this preprint. Click to download.

- Additionalfiles20200501.docx 